\title{
Bringing Electrochemical 3D Printing to the Nanoscale
}

\section{Supporting Information}

Julian Hengsteler, ${ }^{1}$ Barnik Mandal, ${ }^{I}$ Cathelijn van Nisselroy, ${ }^{,}$Genevieve P. S. Lau, ${ }^{2}$ Tilman Schlotter, ${ }^{l}$ Tomaso Zambelli ${ }^{1}$ and Dmitry Momotenko ${ }^{1,3}{ }^{*}$

${ }^{1}$ Laboratory of Biosensors and Bioelectronics, Institute for Biomedical Engineering, ETH Zurich, Zurich, CH-8092, Switzerland

${ }^{2}$ School of Physical and Mathematical Sciences, Division of Chemistry and Biological Chemistry, Nanyang Technological University, 637371, Singapore

${ }^{3}$ Department of Chemistry, Carl von Ossietzky University of Oldenburg, Oldenburg, D-26129, Germany

*dmitry.momotenko@uol.de 


\section{Table of Contents}

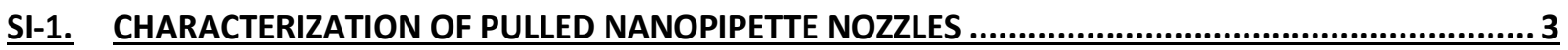

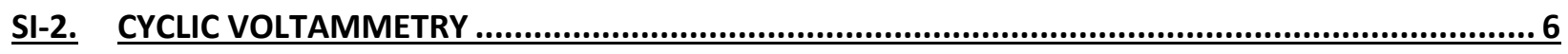

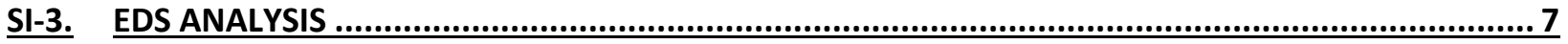

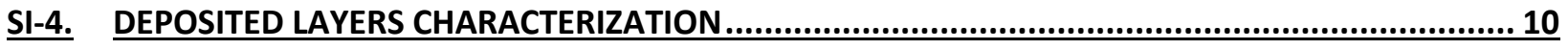

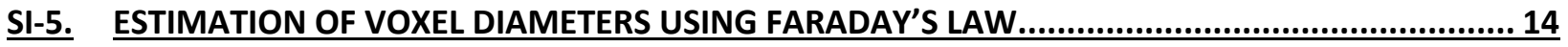

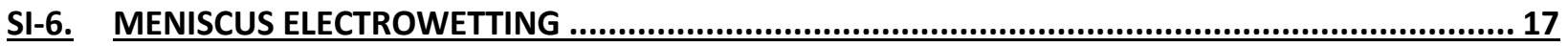

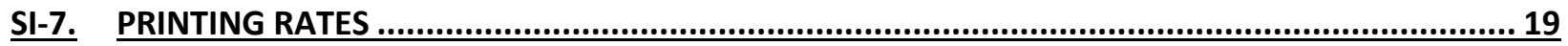

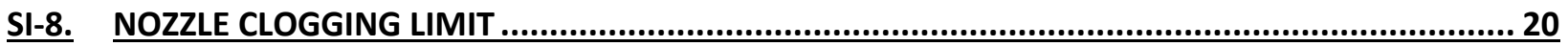




\section{SI-1. Characterization of pulled nanopipette nozzles}

The printing nozzles - nanopipettes were fabricated with a P2000 pipette puller by Sutter Instruments using the parameters listed in Table S1. Their opening sizes were characterized by transmission electron microscopy (TEM). Figure S1 provides example images of the four types of pipettes used in this work. The inner radii of the pipettes in Figure S1c and S1d are $\sim 25 \mathrm{~nm}$ and $\sim 110 \mathrm{~nm}$, respectively, measured using a standard image analysis software (ImageJ, open source).

We also managed to fabricate two types of very small nozzles. One, as exemplified in Figure $\mathrm{S} 1 \mathrm{~b}$, is rather stable even in the electron beam and could be imaged at reasonably high resolution so that the aperture and the inner part of the nanopipette are clearly visible. Its inner radius is $\sim 0.5-$ $1 \mathrm{~nm}$.

The tips of the other nanopipette (Figure S1a) exhibited much less stability in the electron beam and the attempts to image those at high magnification failed as that led to the destruction of the tip region. Although exact determination of the opening from the electron microscope image is impossible, it is clear that the dimensions of the opening should be somewhere in the low single nanometer scale.

To get further information on the opening dimensions of these small nozzles, we tested their electrical conductivity in $2 \mathrm{M} \mathrm{KCl}$ solution. The respective voltammograms are shown in Figure $\mathrm{S} 2$, suggesting that electrical resistance of the nanopipette $R_{\mathrm{p}}$ is around $1.186 \mathrm{G} \Omega$. Assuming the nozzle half-cone angle of ca. $0.7^{\circ}$ (from TEM images) and the diffusion coefficients of potassium and chloride ions $D_{i}$ around $1.98 \mathrm{~m}^{2} \mathrm{~s}^{-1},{ }^{1}$ one can calculate the opening radius according to the following equation ${ }^{2}$

$r=\frac{1}{2 \pi R_{p} \sigma}\left(1+\cot \frac{\alpha}{2}\right)$

where the conductivity $\sigma$ is calculated as the function of salt concentration $c_{0}$ (with $F, R$ and $T$ denoting faraday and gas constants as well as temperature, respectively):

$\sigma=2 \frac{F^{2}}{R T} D_{i} c_{0}$

In result, the nozzle radius can be estimated as $c a .0 .74 \mathrm{~nm}(1.48 \mathrm{~nm}$ diameter). 

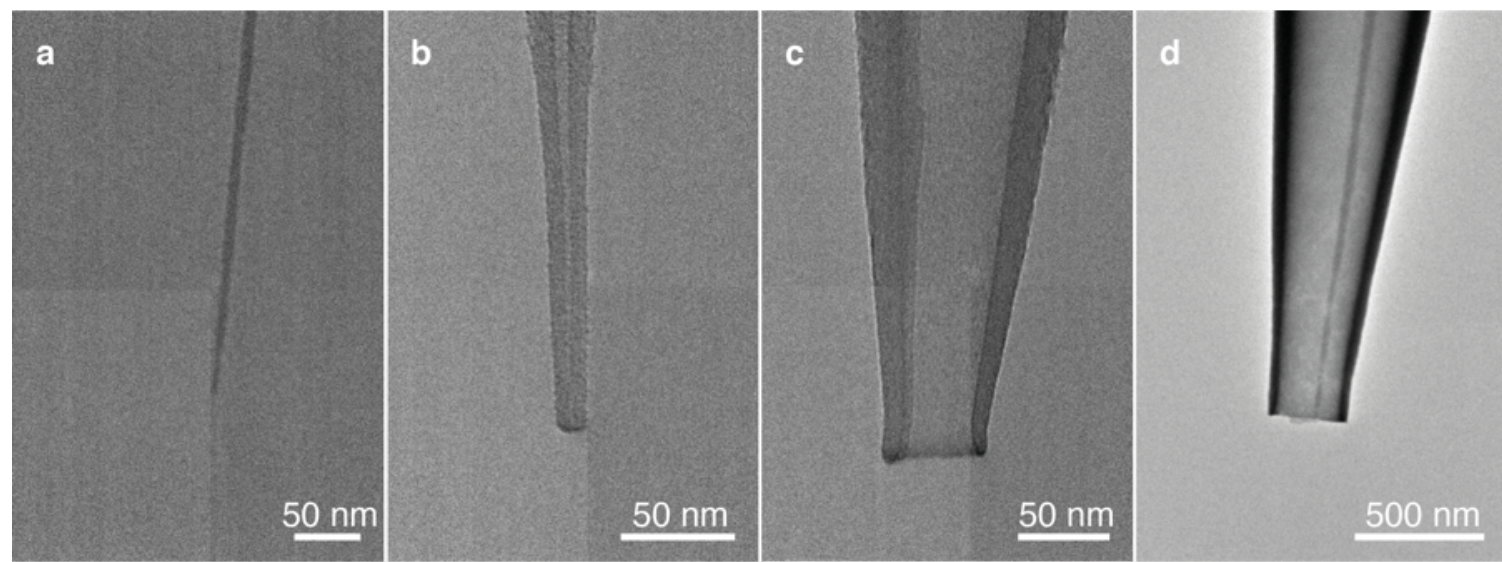

Figure S1. TEM images of the three different types of pipettes used in this work. The opening radii are approximately a) $0.8 \mathrm{~nm}$, b) $0.5-1 \mathrm{~nm}$, c) $25 \mathrm{~nm}$, d) $110 \mathrm{~nm}$.

Table S1: Pulling parameters for pulling the capillaries using a P2000 pipette puller by Sutter Instruments.

\begin{tabular}{lllllll}
\hline Nozzle opening diameter & Line \# & Heat & Filament & Velocity & Delay & Pull \\
\hline $\mathbf{2 2 0} \mathbf{~ n m}$ & 1 & 350 & 3 & 30 & 220 & - \\
& 2 & 350 & 3 & 40 & 180 & 120 \\
$\mathbf{5 0} \mathbf{~ n m}$ & 1 & 350 & 3 & 50 & 220 & - \\
& 2 & 350 & 3 & 40 & 180 & 120 \\
$\mathbf{2} \mathbf{~ n m}$ & 1 & 750 & 4 & 60 & 135 & 180 \\
$\mathbf{1 . 4 8} \mathbf{~ n m}$ & 1 & 725 & 4 & 40 & 145 & 175 \\
\hline
\end{tabular}




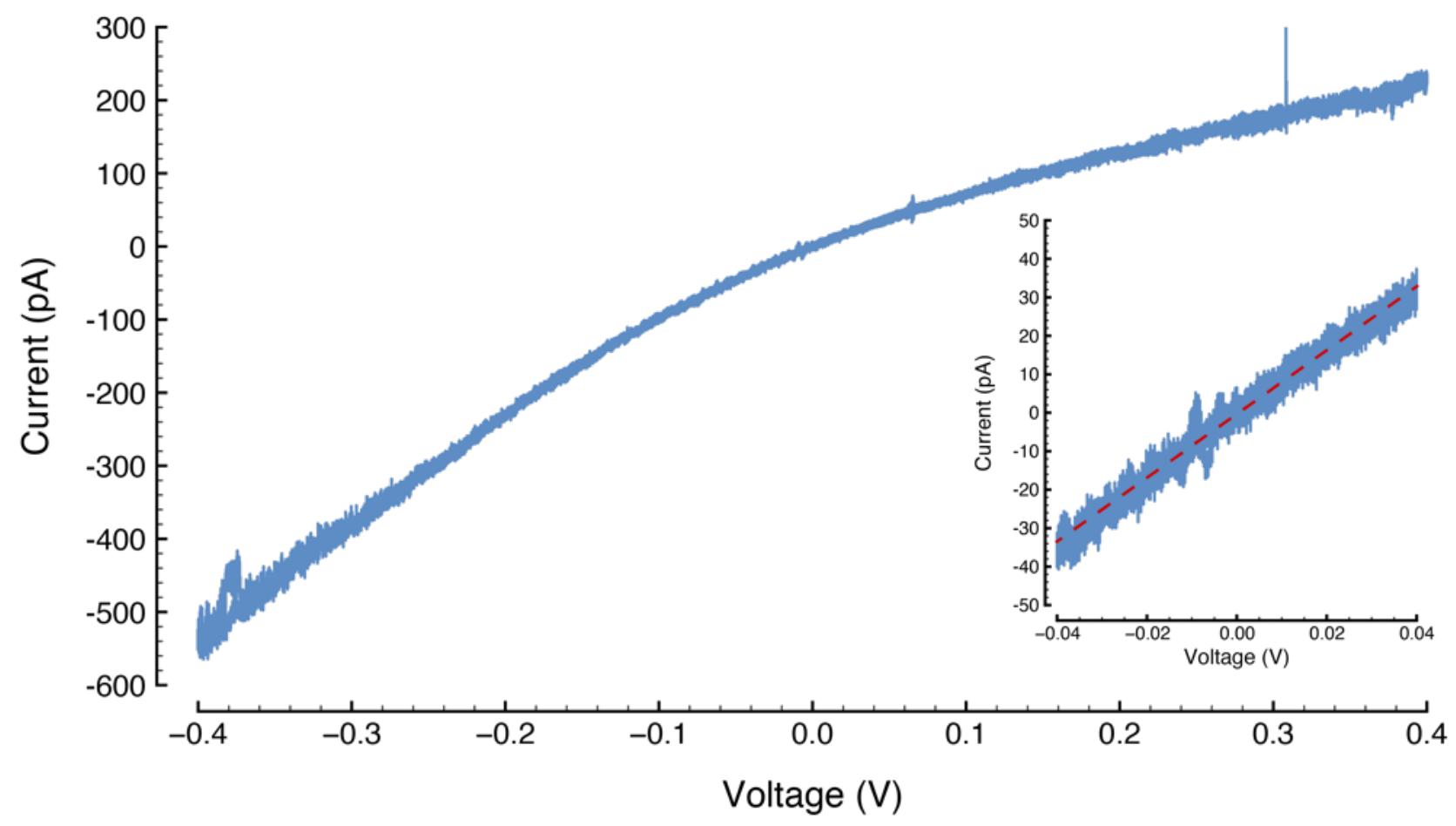

Figure S2. Voltammogram of a nanopipette as in Figure S1a used to estimate its electrical resistance. The measurements were taken in $2 \mathrm{M} \mathrm{KCl}$ solution. From the inverse of the slope of the region zoomed-in in the inset the resistance was calculated to be $1.186 \mathrm{G} \Omega$. 


\section{SI-2. Cyclic voltammetry}

Cyclic voltammetry (CV) was performed to elucidate the choice of deposition potential within this work. Figure S3 shows the resulting current-voltage (IV) curve. The measurement was performed with a $c a .250 \mathrm{~nm}$ nozzle positioned statically above the substrate. An Ag wire was used as QRCE in the pipette, while the gold thin film on the substrate served as WE, similarly to our printing experiments. The pipette was filled with the same electrolyte used for printing experiments $(0.5 \mathrm{M}$ $\mathrm{CuSO} 4$ in $1 \mathrm{M} \mathrm{H} 2 \mathrm{SO} 4, \mathrm{pH}$ 0.4). The scan rate was chosen to be $0.1 \mathrm{~V} \mathrm{~s}^{-1}$ starting in the direction of cathodic potentials. The $\mathrm{CV}$ indicates the onset of $\mathrm{Cu}$ reduction around $-0.29 \mathrm{~V}$, copper dissolution peak is positioned at around $-0.05 \mathrm{~V}$.

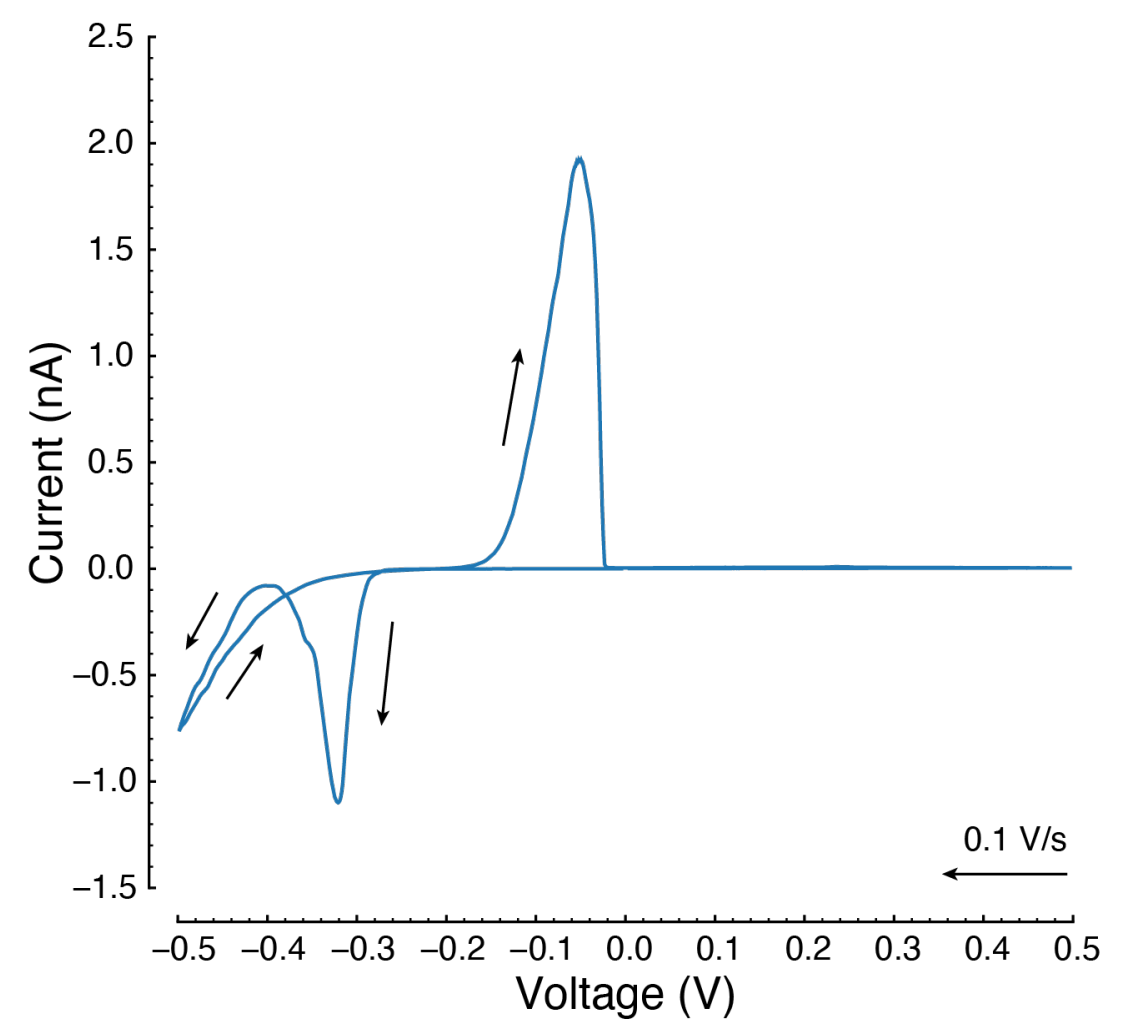

Figure S3. Cyclic voltammogram acquired with a $\sim 250 \mathrm{~nm}$ nozzle forming a meniscus on a gold thin film substrate. The pipette was filled with $0.5 \mathrm{M} \mathrm{CuSO}_{4}$ and an $\mathrm{Ag}$ wire was used as QRCE. The voltage was swept with a rate of $0.1 \mathrm{~V} \mathrm{~s}^{-1}$. The onset of $\mathrm{Cu}$ reduction is measured at $-0.29 \mathrm{~V}$. Arrows on the plot indicate sweep direction. 


\section{SI-3. EDS analysis}

Energy-dispersive X-ray spectroscopy (EDS) was performed to analyze the chemical composition of the printed material. Therefore, an array of $6 \times 6,4 \mu \mathrm{m}$ tall pillars was printed with a $\sim 250 \mathrm{~nm}$ pipette tip, at $-0.35 \mathrm{~V}$ and a current threshold of $-40 \mathrm{pA}$. EDS maps were taken from the top as well as at a tilt of $45^{\circ}$. The results are shown in Figure S4. The secondary electron image from the SEM is shown in Figure S4a illustrating the geometry of the features. Figure S4b shows the image taken with the backscatter electron detector. The strong contrast between the pillars and the substrate shows that the substrate consists of heavier elements (stronger backscattering) than the darker pillars (weaker backscattering).

In Figure S4c an intensity map of the two most distinctive phases as detected by the EDS software (Team, EDAX) is shown. Figure S4d shows the energy spectra of these two phases matching the colors of the intensity map in Figure S4c. One distinct intensity peak can be observed for each of the two phases - $\mathrm{Si}-\mathrm{K} \alpha$ (top, blue phase) and $\mathrm{Cu}-\mathrm{L} \alpha$ (bottom, red phase), highlighting the purity of the printed $\mathrm{Cu}$ features. The intensity maps of these two energy levels are overlayed in Figure S4e (blue: $\mathrm{Si}-\mathrm{K} \alpha$, red: $\mathrm{Cu}-\mathrm{L} \alpha$ ). Additionally, the respective intensities along the dotted line in Figure S4e are shown in Figure S4f. Overall a higher intensity level of Si-K $\alpha$ can be observed. This can be explained by the large interaction volume of the electron beam, penetrating through the pillars, rendering them partially transparent. 

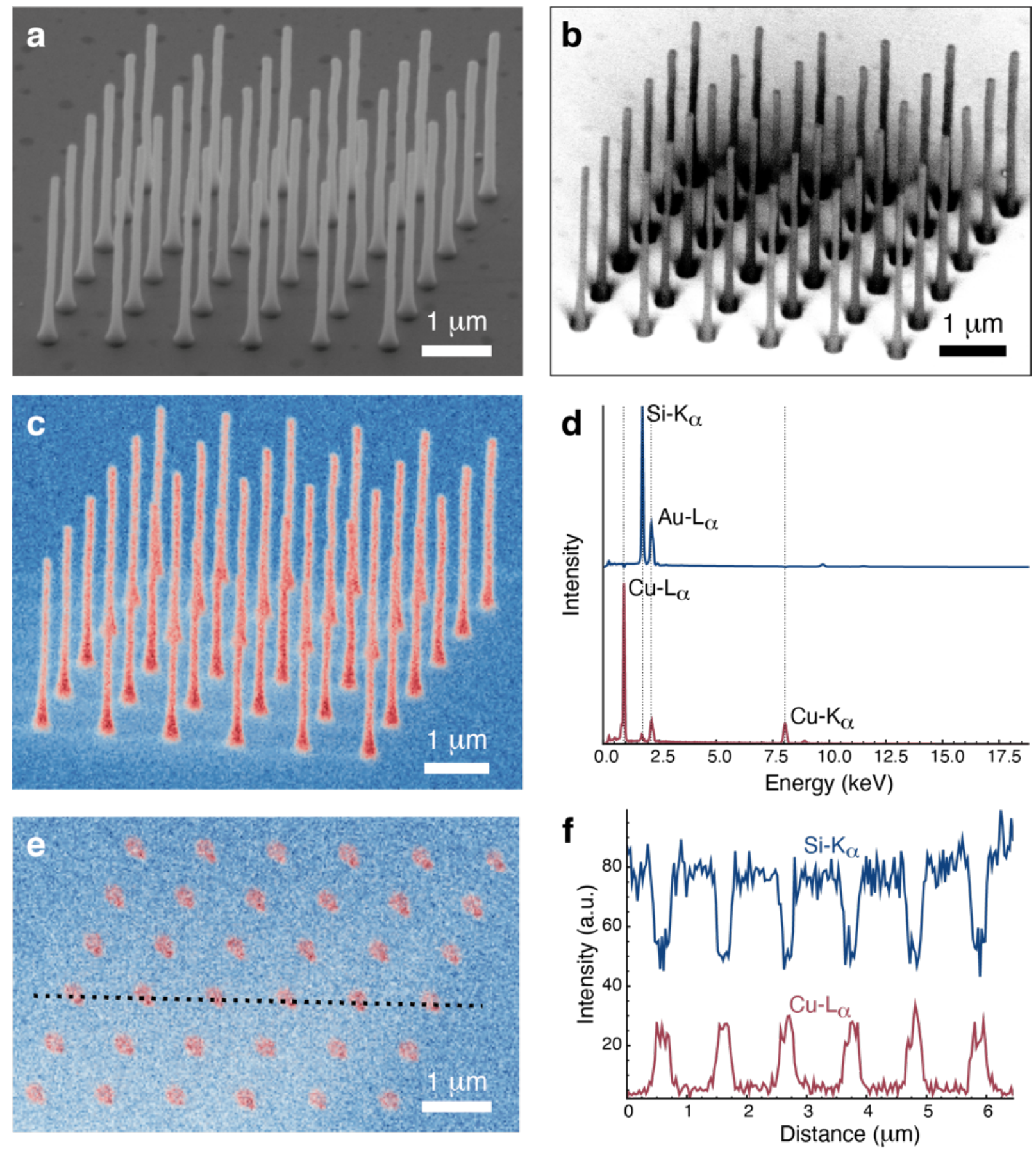

Figure S4. SEM and EDS analysis of the 3D printed materials. The analyzed structures are shown in the SEM image in a) and were printed with a $\sim 250 \mathrm{~nm}$ pipette, at $-0.35 \mathrm{~V}$ and a current threshold of -40 pA. b) Shows the backscatter electron image. The bright background indicates the heavier materials ( $\mathrm{Si} \& \mathrm{Au}$ ) of the substrate as compared to the dark pillars consisting of $\mathrm{Cu}$. c) The EDS phase map separating the two most distinctive material phases, detected by the X-ray detector. d) 
The energy spectra corresponding to the two phases shown in c). e) An overlay of the two intensity maps of the energy levels of interest $\mathrm{Si}-\mathrm{K} \alpha$ (blue) and $\mathrm{Cu}-\mathrm{L} \alpha$ (red). f) The X-ray intensities of Si$\mathrm{K} \alpha$ (blue) and $\mathrm{Cu}-\mathrm{L} \alpha$ (red) across the black, dotted line in e). 


\section{SI-4. Deposited layers characterization}

The thickness of the deposited layer depends on the charge transferred in the deposition period and the lateral confinement due to the meniscus boundaries. Figure S5a shows the influence of the applied deposition voltage on printed layer thickness. At an applied voltage of $-0.55 \mathrm{~V}$ the average layer thickness was $10.7 \mathrm{~nm}( \pm 1.1 \mathrm{~nm})$ whereas at a lower voltage magnitude of $-0.35 \mathrm{~V}$ the layer thickness decreased to $3 \mathrm{~nm}( \pm 0.1 \mathrm{~nm})$. The exponential increase with increasing voltage magnitude can be explained by the increasing electromigration of species towards the interface at higher voltage magnitudes as well as faster electrode kinetics in accordance with Butler-Volmer formulation. Figure S5b shows the dependence of the layer thickness on the set-point of the threshold current that was used to mark the onset of a liquid meniscus as a feedback mechanism. With a current threshold of $-30 \mathrm{pA}$ the average layer height was $10.8 \mathrm{~nm}( \pm 1.6 \mathrm{~nm})$ decreasing down to $5 \mathrm{~nm}( \pm 4.9 \mathrm{~nm})$ at $-5 \mathrm{pA}$.

a

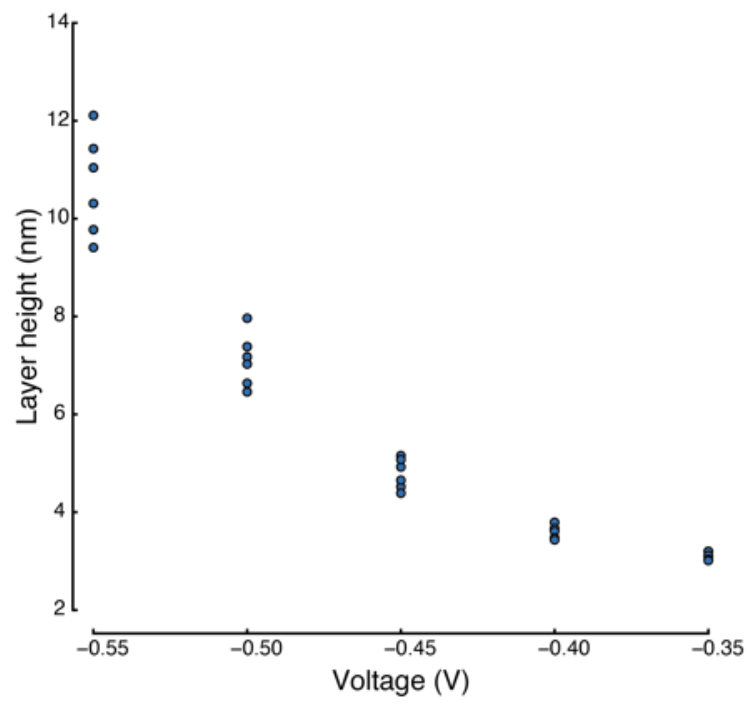

b

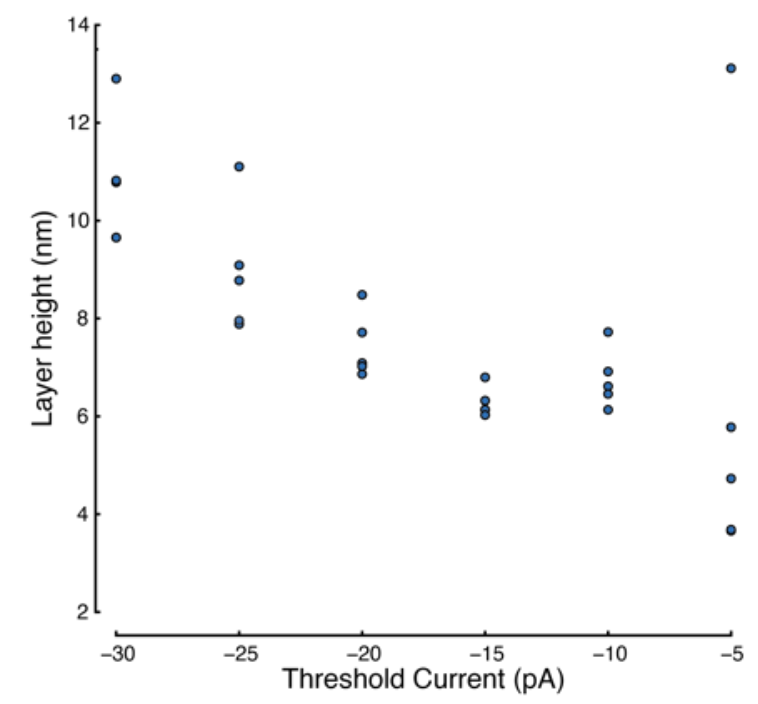

Figure S5. The average deposited layer heights for each pillar printed at different voltages and a fixed threshold current of -4 pA (a) or with different current thresholds at a fixed voltage of -0.35 $\mathrm{V}(\mathrm{b})$. 
The shape and topography of single to a few deposited layers was investigated by atomic force microscopy (AFM). A array of 50 columns and 4 rows was printed with a $\sim 50 \mathrm{~nm}$ pipette tip, at $0.4 \mathrm{~V}$ and a current threshold setpoint of $-30 \mathrm{pA}$. In the first row only one single layer was deposited, in the second row a stack of two layers, in the third four layers and in the fourth a total of 8 layers was deposited. Afterwards an area of about 4.3 by $4.3 \mu \mathrm{m}$ was scanned with the AFM (Head: FlexFM 5; Controller: C3000, both Nanosurf AG, Switzerland; Tip: PPP-NCLR-10, Nanosensors, Switzerland). The results are shown in Figure S6. In Figure S6a the scanned area is shown with single layers marked by the light blue rectangles and the 8 layered deposits marked by the red rectangles. The scan shows that the initial layers are not perfectly round but can be elongated or elliptic. Also, the diameters of the pillars base, formed by the first layers, is evidently larger than found in the pillar parts further away from the substrate.

To determine the heights of the different structures each of the rectangles was separately offset such that the mean of the noisy background signal in the rectangle is placed at zero. Afterwards the maximum value was found and taken as the height. Figure S6b shows the average height of the corresponding group with the error bars representing the $95 \%$ confidence interval within the respective group. The first layer is thicker $(20.72 \pm 2.29 \mathrm{~nm})$ than the consecutive ones, probably because printing of the first layer occurs on a different material $(\mathrm{Au})$ than consecutive layers on printed $\mathrm{Cu}$. The average layer heights estimated from the AFM measurements, with a total average height of $35.13 \mathrm{~nm}( \pm 4.36 \mathrm{~nm})$ for 8 layers, hence on average $4.39 \mathrm{~nm}$ per single layer correlate reasonably well with the measurements directly from the print data (taking into account variation of the layer thickness depending on voltage and threshold current). Figure S7a shows the zoomedin view of a single deposited layer ( $2^{\text {nd }}$ from the left of top row of S6a). The graph in S7b shows the height profile taken along the pink line in Figure S6c. Similarly, in Figure S7c a zoomed-in 
view of an 8-layer structure ( $2^{\text {nd }}$ from the left of bottom row) is shown with the height profile along the pink line shown in Figure S7d.
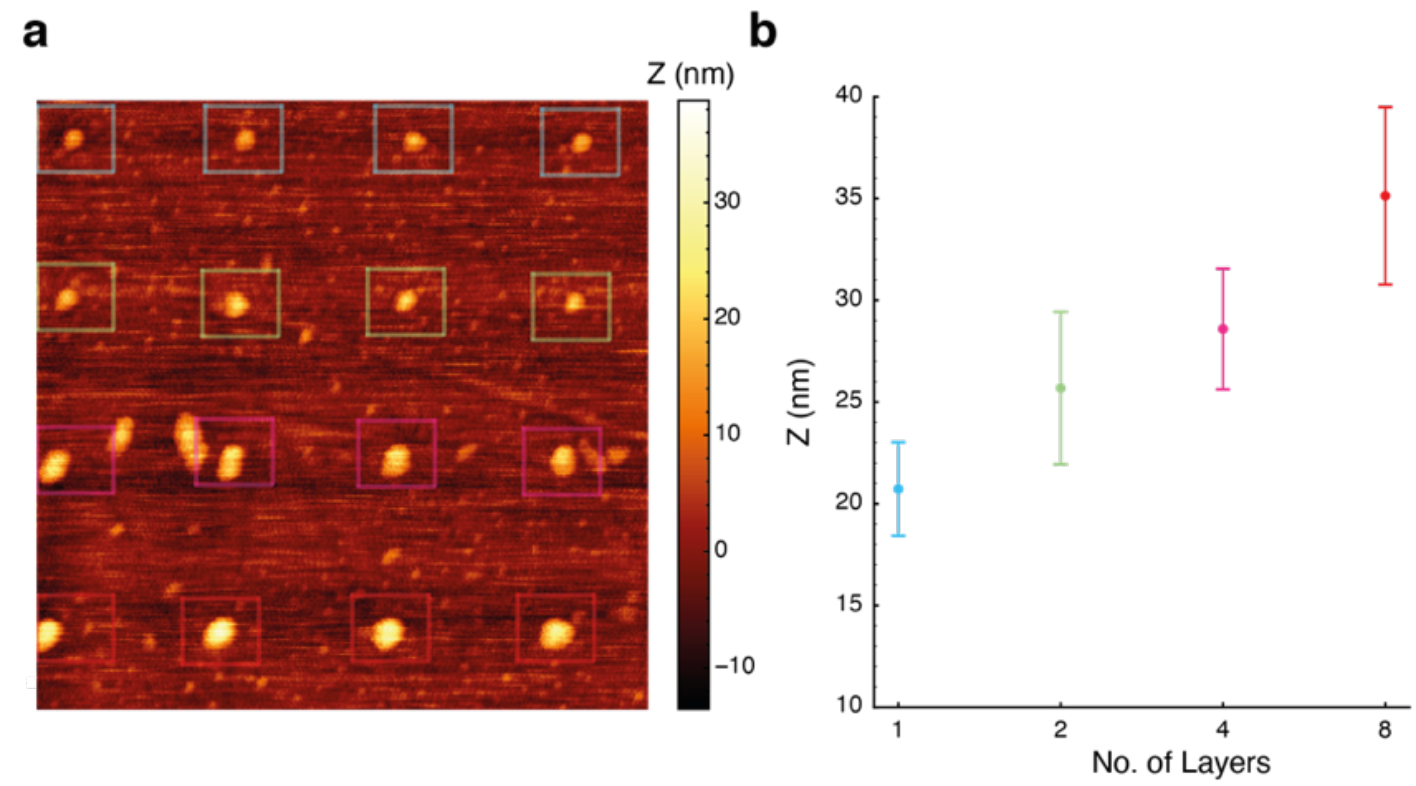

Figure S6. AFM analysis of single deposited layers. The structures were deposited with a $\sim 50 \mathrm{~nm}$ pipette at $-0.4 \mathrm{~V}$ and a current threshold setpoint of $-30 \mathrm{pA}$. a) Scan of a $4.3 \times 4.3 \mu \mathrm{m}$ section of an array of 1-, 2-, 4- and 8-layer deposits, marked by the light blue, green, pink, and red rectangles, respectively. b) The average height of the respective deposits taken from the maximum values of the area marked by the rectangles after zeroing the mean of the noisy signal from the substrate. The error bars represent the upper and lower limit of the $95 \%$ confidence interval. 

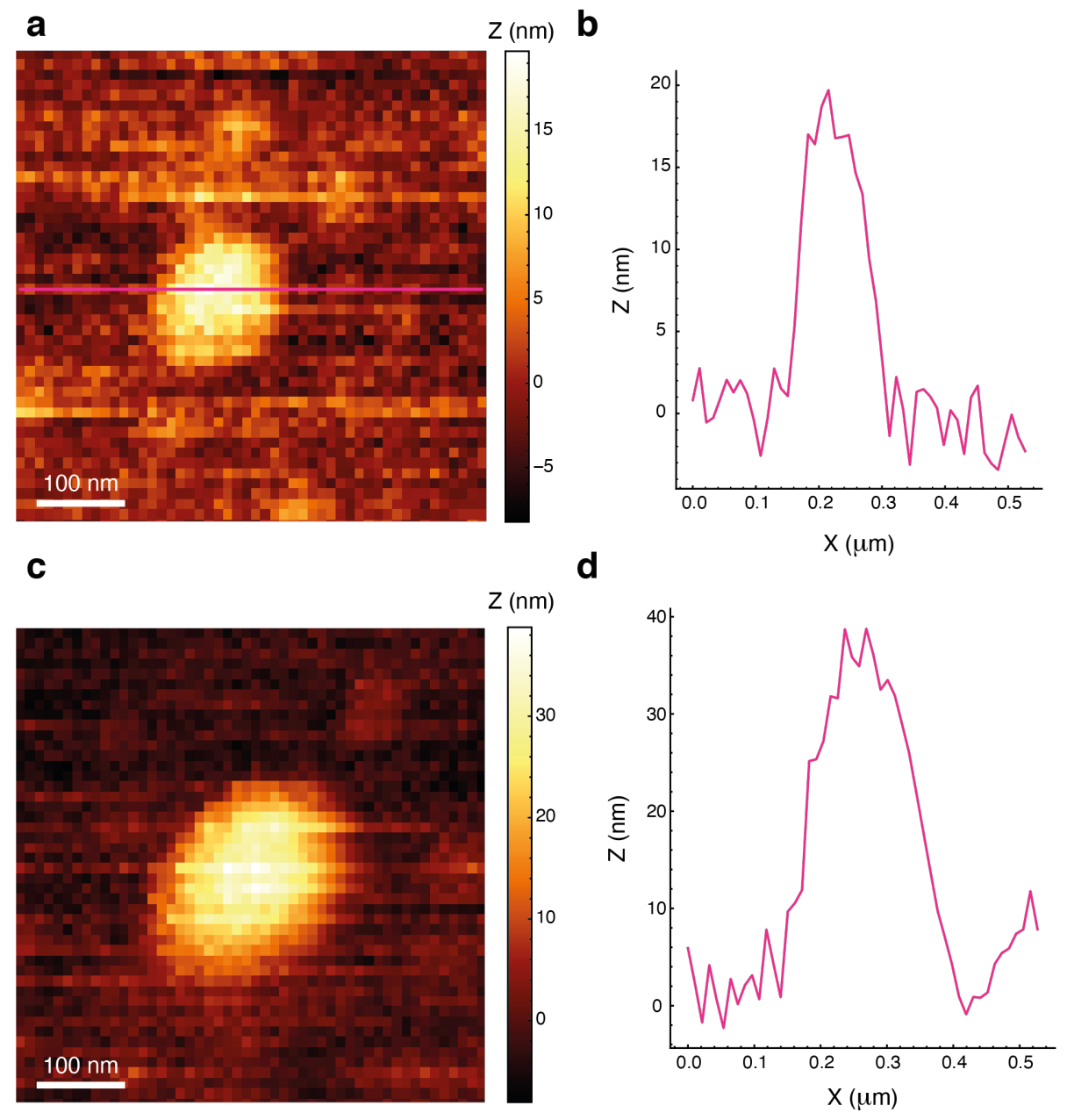

Figure S7. Zoom-in on the AFM scan shown in Figure S6a along with height profiles taken across the pink lines. $(a, b)$ show the scan taken from a single layer similarly $(c, d)$ show the same representation of an 8 layer deposit. 


\section{SI-5. Estimation of voxel diameters using Faraday's law}

To approximate the diameter of a single deposited layer Faraday's Law of electrolysis was used:

$m=\frac{Q M}{F n}$

with $m$ the mass of the deposit, $Q$ the transferred charge, $M$ the molar mass of the deposited material, $F$ the Faraday constant (96 $485.3329 \mathrm{~A} \mathrm{~s} \mathrm{~mol}^{-1}$ ) and $\mathrm{n}$ the valency of the ions involved in the reaction. The mass of a cylindrical deposit is:

$m=V * \rho=A * h * \rho=\pi * r^{2} * h * \rho$

with the height of the deposit $h$ and $r$ its radius. The charge $Q$ can be calculated by integrating the current during deposition:

$Q=\int_{t 1}^{t 2} I d t$

Assuming a constant meniscus height at landing the height of one deposition can be calculated from the piezo displacement values of the vertical actuator from two consecutive landings. Equations eq. S3 - S4 then yield the diameter $d$ of the single layer:

$d=2 * \sqrt{\frac{m}{\pi h \rho}}=2 * \sqrt{\frac{Q M}{\pi F n h \rho}}$

Table S2 summarizes the values used in this work to calculate the diameter. As shown in Figure $2 \mathrm{~b}$ (main text) the calculation provides an underestimate (typically $\sim 20 \mathrm{~nm}$ ) of the real feature size, most likely due to the relatively low signal-to-noise ratio in the measured current and errors arising from its integration. Furthermore, the feature size estimation could be affected by at least two other factors, namely: i) response time of the current amplifier (in our case, $300 \mu$ s are needed for $10 \%$ $90 \%$ amplification); ii) a relatively slow data acquisition $(\sim 2 \mathrm{kHz})$. Considering that the typical meniscus residence time on the substrate is only a couple of milliseconds, the errors may arise due to a low number of data points in each printing cycle; and iii) porosity of the printed structures, hence a different density than that expected from the bulk value of copper. Despite this slight discrepancy between the estimated and the real feature size, however, both trends in Figure $2 \mathrm{~b}$ demonstrate that the feature size increases linearly with the increase of the cathodic potential. To elucidate the possible reason for the discrepancy, we printed $\mathrm{Cu}$ pillars with a slightly larger 
nozzles of $\sim 250 \mathrm{~nm}$ diameter. For a larger aperture the expected meniscus size increases, thus the current peaks are not only higher in magnitude (higher SNR) but also broader (it takes longer to break a larger meniscus). This way more samples are taken during data acquisition and integration of the current, to yield the transferred charge, is more accurate. In addition, the printed pillars are larger and focused ion beam (FIB) milling can be used to reveal the inner morphology of the asprinted features to provide information on the porosity of the metal. Three $3 \mu \mathrm{m}$ tall pillars were printed at $-0.35 \mathrm{~V},-0.4 \mathrm{~V},-0.45 \mathrm{~V},-0.5 \mathrm{~V}$ and $-0.55 \mathrm{~V}$, respectively. Figure S8 summarizes the results of the described experiment. Figure S8a shows the comparison of the measured diameters from SEM images (Figure S8c) vs the estimation from Faraday's law. The trend shown is similar to the one shown in Figure $2 \mathrm{~b}$ (main text) for pillars printed with smaller nozzle apertures. At higher deposition voltage magnitudes the diameter increases. As expected, at this scale the estimated and measured values for the diameters match better. This is due to the increased number of current samples (broader peaks) at each printing cycle and improved signal-to-noise ratio (larger current signals). Both of these factors contribute to improved estimation of feature size directly from the printing data. Figure S8b shows an example of a peak taken from the pillar printed at $0.45 \mathrm{~V}$ with the recorded samples marked in red. This clearly shows the increased SNR at these scales and the larger number of samples for one peak. Figure S8c shows a whole pillar for each potential next to a pillar, from the same set, with a FIB milled top part. The milled regions reveal the inner morphology of the as-printed metal. This shows that only few small (typically, only several nm across) voids are formed during deposition, which hardly can account for the mismatch between the Faraday's law and SEM measurement.

Table S2. Material parameters for copper

\begin{tabular}{l|l} 
Parameter & Value \\
\hline $\boldsymbol{\rho}$, Cu density & $8.96 \mathrm{~g} \mathrm{~cm}^{-3}$ \\
$\boldsymbol{n}$, charge number & 2 \\
$\boldsymbol{M}$, Cu molar mass & $63.546 \mathrm{~g} \mathrm{~mol}^{-1}$
\end{tabular}




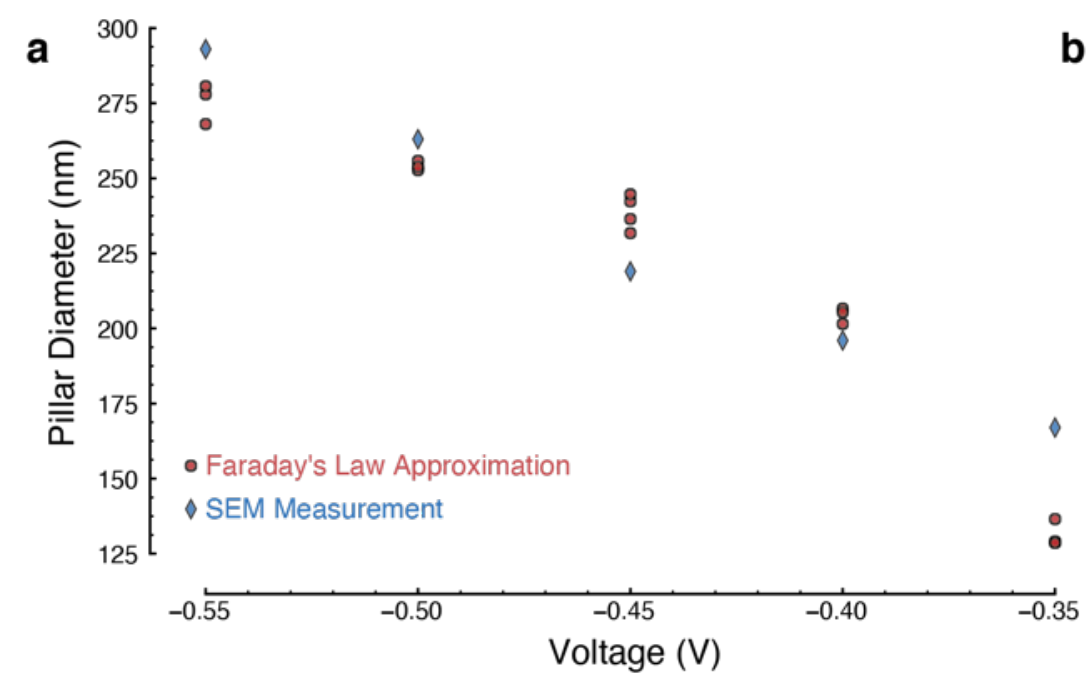

b

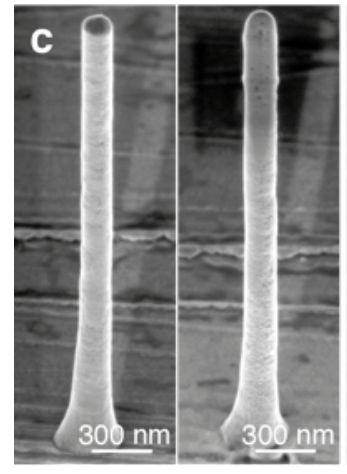

$-0.35 \mathrm{~V}$

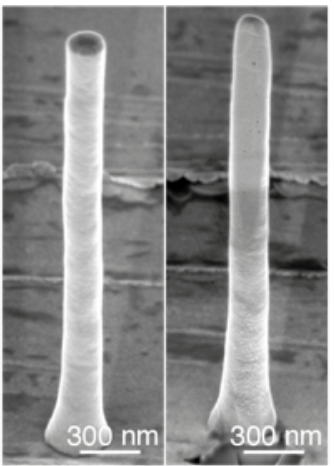

$-0.4 \mathrm{~V}$

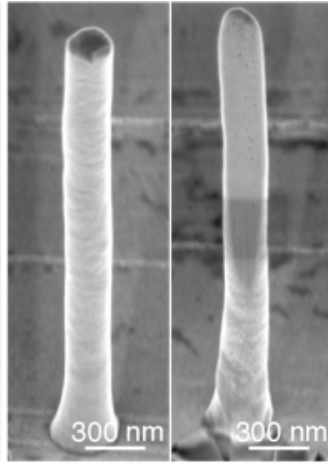

$-0.45 \mathrm{~V}$

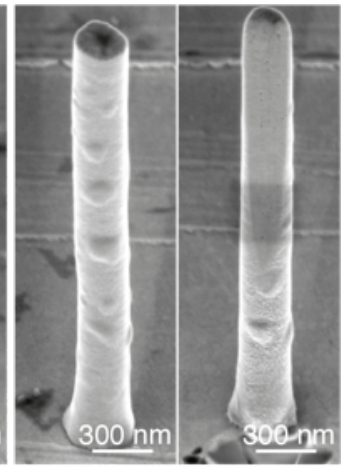

$-0.5 \mathrm{~V}$

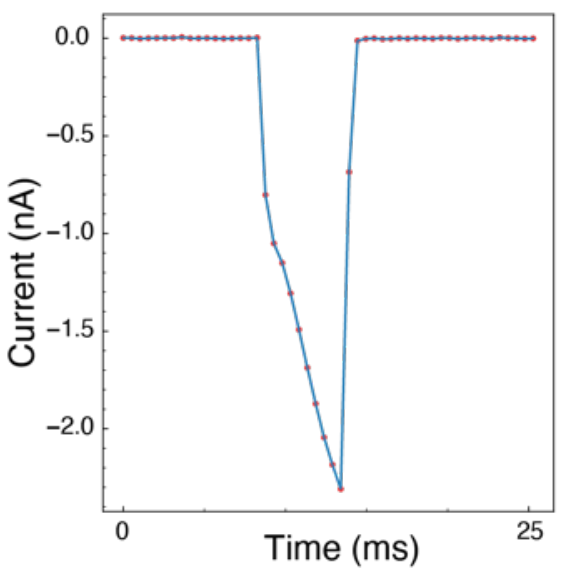

Figure S8. Results from printing with a ca. $250 \mathrm{~nm}$ aperture. a) The diameters taken from the SEM images and calculated from the raw data using Faraday's law as a function of deposition potential. b) An individual printing cycle from the print at $-0.45 \mathrm{~V}$ with the data values highlighted by the red marker points. c) SEM images ( $45^{\circ}$ tilt) of single pillars printed with a ca. $250 \mathrm{~nm}$ aperture at different deposition voltages. The left images show the as-printed pillar, the right images show pillars with a FIB-milled tip. 


\section{SI-6. Meniscus electrowetting}

To study the effect of different voltages on the meniscus formation optical measurements were performed. Unpulled quartz capillaries (ID $0.5 \mathrm{~mm}$, OD, $1 \mathrm{~mm}$, QF100-50-10, Friedrich \& Dimmock) were filled with the $\mathrm{CuSO}_{4}$ electrolyte, used for printing. A silver wire was placed inside of the capillaries and a voltage applied between the wire and the Au coated glass substrate (same as the substrates for printing experiments). The capillaries were slowly approached to the substrate and as soon as a current higher than the noise floor was measured retracted by $500 \mu \mathrm{m}$. A picture was taken with a microscope camera (AM7915MZTL - EDGE, Dino Lite) as soon as a stable meniscus was formed. This was repeated for $-0.35 \mathrm{~V},-0.45 \mathrm{~V}$ and $-0.55 \mathrm{~V}$. Figure $\mathrm{S} 9 \mathrm{a}$ shows the images of the menisci at the different potentials. For higher voltage magnitudes a larger contact angle at the liquid-substrate-air interface can be observed. At $-0.35 \mathrm{~V}$ the contact angle sets at about $55^{\circ}$, at $-0.4 \mathrm{~V} 64^{\circ}$ were measured and the largest angle of $70^{\circ}$ at $-0.55 \mathrm{~V}$. These results support the hypothesis of ultralow voltage electrowetting causing larger meniscus footprint (and larger feature diameter) at higher cathodic potentials. In Figure S9b an overlay of the two extreme cases $(-0.35 \mathrm{~V}$ and $-0.55 \mathrm{~V})$ is shown with the contours of the menisci marked in red $(-0.35 \mathrm{~V})$ and yellow $(-0.55 \mathrm{~V})$. The transparent grayscale image shows the meniscus of the meniscus at $-0.35 \mathrm{~V}$ that is clearly smaller than the colored image in the background of the meniscus at $-0.55 \mathrm{~V}$.

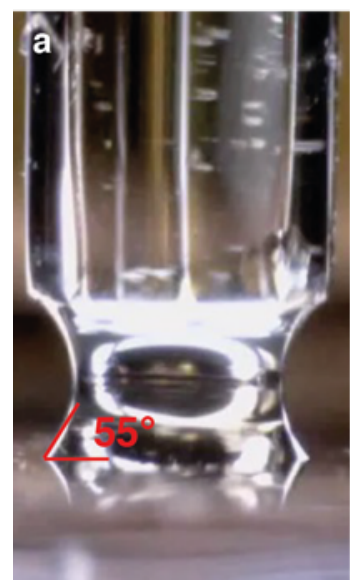

$-0.35 \mathrm{~V}$

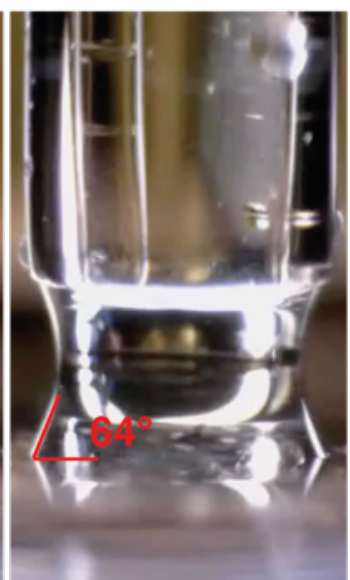

$-0.4 \mathrm{~V}$

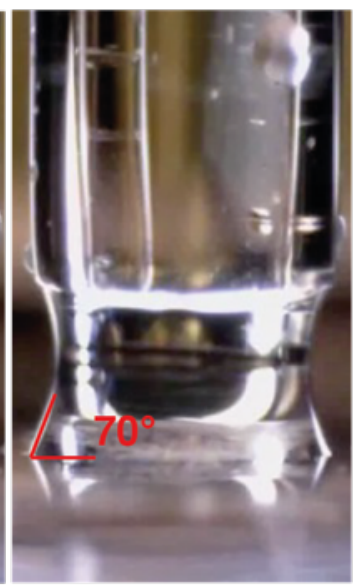

$-0.55 \mathrm{~V}$

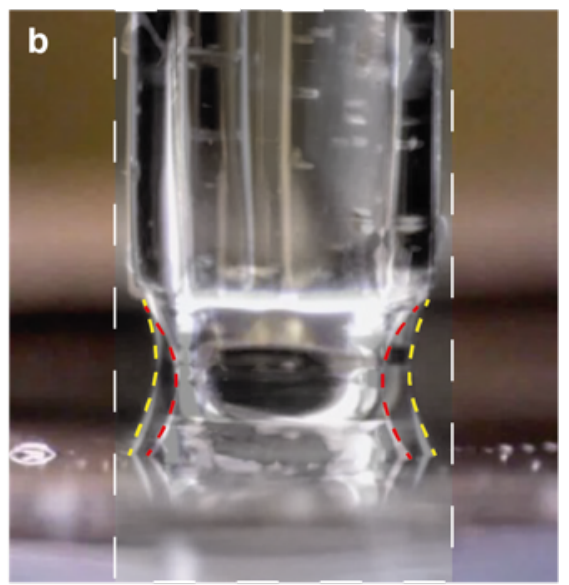

Figure S9. Images of menisci formed at the end of an unpulled capillary at different voltages. a) Three menisci taken at three different voltages (voltage value displayed on the images). The contact angle increases with increasing cathodic potential. b) Overlayed image of the meniscus formed at $-0.35 \mathrm{~V}$ (transparent, grayscale) on top of the image of the meniscus at $-0.55 \mathrm{~V}$ (colored). 
The two menisci are marked by the dashed lines in red and yellow for $-0.35 \mathrm{~V}$ and $-0.55 \mathrm{~V}$, respectively. 


\section{SI-7. Printing rates}

As discussed, higher currents indicate a higher amount of charge transferred at the interface, hence more material deposited. In result, the applied voltage not only influences the diameters of the deposited individual layers but also their thicknesses (SI-4). This results in a change in the printing speed. The average printing rates approach $6.6\left( \pm 1.1 \mathrm{~nm} \mathrm{~s}^{-1}\right), 12.5\left( \pm 2.0 \mathrm{~nm} \mathrm{~s}^{-1}\right), 24.4( \pm 2.5 \mathrm{~nm}$ $\left.\mathrm{s}^{-1}\right), 44.8\left( \pm 4.6 \mathrm{~nm} \mathrm{~s}^{-1}\right)$, and $69.7\left( \pm 7.4 \mathrm{~nm} \mathrm{~s}^{-1}\right) \mathrm{nm} \mathrm{s}^{-1}$, for $-0.35,-0.4,-0.45,-0.5$ and $-0.55 \mathrm{~V}$, respectively (Figure S10). The printing rate exhibits an exponential increase at larger cathodic potentials as could be expected from a Butler-Volmer relation.

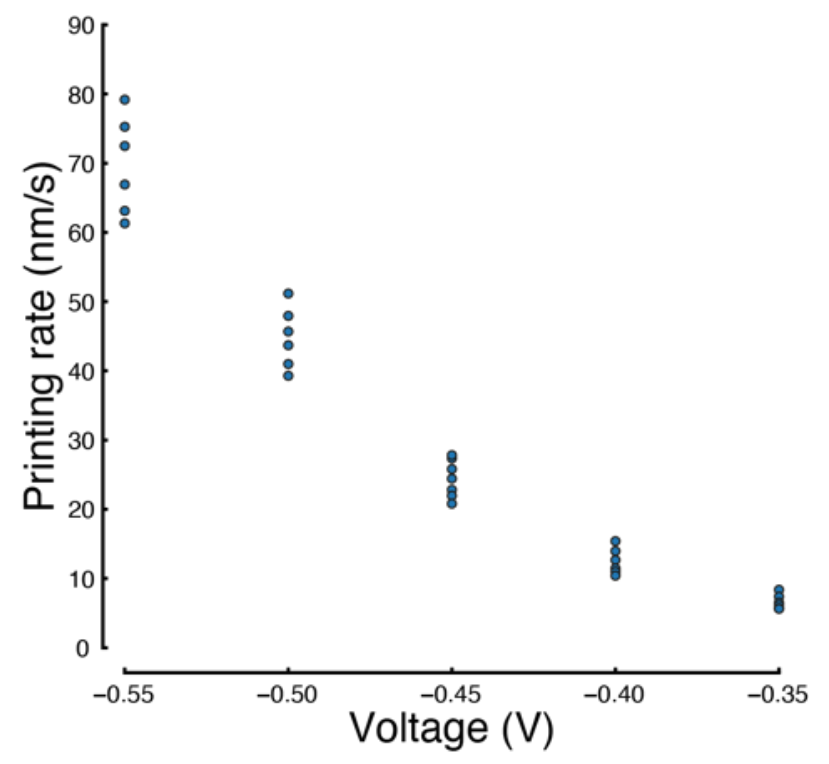

Figure S10. The average growth rate for pillars printed at different voltages. 


\section{SI-8. Nozzle clogging limit}

When the height of a single layer - printed in one time window of deposition - is larger than the meniscus it will grow into the nozzle and clog it. Rearranging equation (S6) to yield the layer height and assuming the meniscus height as well as the radius of the deposit to equal the radius of the nozzle opening one can estimate the limit for the nozzle diameter $d_{\text {limit }}$ as follows:

$$
\frac{Q * M}{\pi \rho F n\left(\frac{d_{\text {limit }}}{2}\right)^{2}}-\frac{d_{\text {limit }}}{2}=0
$$

hence

$d_{\text {limit }}=\left(\frac{Q M}{\pi \rho F n}\right)^{1 / 3}$

with $Q=I * t=3 p A * 3 m s=9 \mathrm{fC}$ this yields $d_{\text {limit }} \cong 9.5 \mathrm{~nm}$.

With similar assumptions one can easily calculate the height of the single printed layer $h$ (by rearranging equation (S6):

$h=\frac{4 M Q}{\pi n \rho F d^{2}}$

For the nozzle with diameter $d$ of $25 \mathrm{~nm}$ the height is $c a .0 .6 \mathrm{~nm}$, while it quickly increases to $164.5 \mathrm{~nm}$ for a nozzle with opening of $1.6 \mathrm{~nm}$ due to an exponential dependence of $h$ with $d$. 


\section{REFERENCES}

(1) Chang, Y. C.; Myerson, A. S. The Diffusivity of Potassium Chloride and Sodium Chloride in Concentrated, Saturated, and Supersaturated Aqueous Solutions. AIChE J. 1985, 31 (6), 890-894. https://doi.org/10.1002/aic.690310603.

(2) Momotenko, D.; Cortés-Salazar, F.; Josserand, J.; Liu, S.; Shao, Y.; Girault, H. H. Ion Current Rectification and Rectification Inversion in Conical Nanopores: A Perm-Selective View. Phys. Chem. Chem. Phys. 2011, 13 (12), 5430-5440. https://doi.org/10.1039/C0CP02595J. 